\title{
Categories, subcategories, and the attenuation of proactive inhibition in free recall
}

\author{
W. P. BROWN and J. T. O. ATKINSON \\ Queen's University, Belfast, Northern Ireland BT7 $1 N N$
}

\begin{abstract}
In two experiments, "proactive inhibition" was observed in the free recall of a succession of 15-word lists. Recall performancc declined across lists when all lists were drawn from the same taxonomic category (e.g., occupations). But this decline did not appear when the successive lists were drawn from discrete subcategories (such as "professional" and "skilled manual" occupations). The findings clarify the conditions in which proactive inhibition effects in free recall are likely to occur. Specifically, when the set of items on any one list is more homogeneous than the set of items from all lists taken together, "proactive inhibition" will be attenuated.
\end{abstract}

When Ss are tested on a succession of memory tasks, performance typically shows a decline across tasks, a phenomenon termed proactive inhibition (PI). At least part of this effect can be shown to depend upon similarities among the memory materials on successive tasks, since switching to a task involving "different" material may lead to a sharp improvement in memory performance. For example, Wickens and Clark (1968) presented three lists of high-evaluation words followed by a list of low-evaluation words; recall performance declined over Lists 1-3 but recovered on List 4 . This "release from PI" presumably occurred because List 4 differed from the others in evaluative meaning, despite the fact that all four lists were similar in other respects, e.g., that all items were words. Note that Reutener (1972) has shown that "word-ness" is a sufficient basis of similarity for the build-up of PI, with release from PI occurring when a switch is made to a list of numbers. What, then, determines whether or not a given similarity between lists will lead to PI effects?

Wickens (1970) supposes that each item presented is encoded in memory in terms of all its attributes or features (structural, semantic, syntactic, etc.) in turn, starting with the most specific and proceeding to the most general. The sequence of encodings is thought to move upward through a hierarchical organization of features. The earliest (lowest, most specific) encodings are of features that discriminate among items within a list; the later (higher, more general) encodings are of features shared by all items on the list. The condition necessary for the build-up of PI seems to be that the features shared within a list should also be shared between lists. But release from PI may be obtained by using a list that retains many of these shared features, so long as it does not retain them all. This suggests that it is the lowest shared feature that is functional in promoting the PI effect: If the lowest within-list shared feature does not coincide with the lowest between-list shared feature, PI effects will not be obtained, no matter how many higher features are shared across lists. The present experiments were designed to test this hypothesis.

\section{COMPILATION OF STIMULUS LISTS}

Experiment I required a pool of 75 names of occupations, which was compiled as follows. The 36 occupations most frequently mentioned in locally collected category norms were given to 30 students who were asked to sort them into groups. Five strong clusters were identified in the groupings, and two occupations were chosen to represent the "core" of each cluster. These five pairs were presented to 12 judges, together with a list of about 75 occupations that they were asked to allocate to one of the five "cores" (or to discard); interjudge agreement was extremely high. In this way, five well differentiated sets of occupation names were obtained, 15 items from each set being used in the experiment.

The 75 items were arranged into five 15 -word lists in two different ways. Each experimental list consisted of the 15 occupations from one of the five sets. The lowest between-list shared feature was assumed to be "occupations," but each list had a lower (more specific) within-list shared feature; these might be labeled "medical," "professional," "shop-trader," "skilled," and "unskilled." The control lists were formed by taking three items from each of the experimental lists. For the control lists, the lowest within-list shared feature was assumed to be identical to the lowest between-list shared feature, i.e., "occupations." For each list, five different item orders were prepared and subsequently used with equal frequency in the experiment.

Experiment II required a pool of 60 names of animals, 15 in each of four well differentiated sets. The items were chosen by a procedure similar to that employed for occupations. The 60 items were arranged into four experimental lists and four control lists. The lowest within-list shared features for the experimental lists might be labeled "farm animals," "small wild creatures (mostly native to Britain)," "large exotic carnivores," and "large exotic herbivores." 
Table 1

Number of Words Recalled from Secondary Memory: Means and Standard Deviations

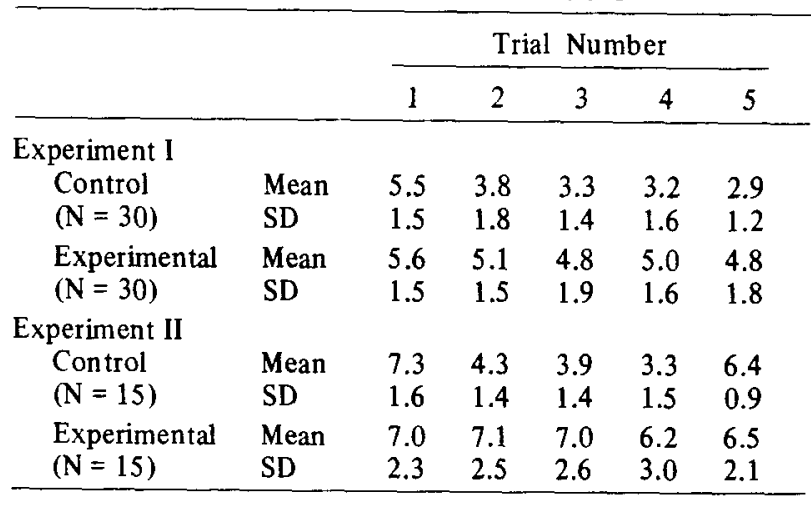

\section{EXPERIMENT I}

Design

The Ss were presented with five 15 -item lists for immediate free recall. Experimental Ss received the experimental lists, control Ss the control lists. Each list was used equally frequently in each of the five serial positions; the order of items within a list was randomized, a total of five different randomized orders being used with each list. Half of the $\mathrm{S} s$ in each group were told that they would hear five lists of words; the other half were told that they would hear five lists of occupations. (Instructions were varied in this way to see whether the salience given to the lowest between-list shared feature by explicitly labeling it for Ss would have an effect.)

\section{Subjects}

There were $60 \mathrm{Ss}$, senior pupils (modal age 17 years) at a local grammar school, 15 in each of the four treatments (Lists by Instructions). They were tested in groups of three.

\section{Procedure}

Details of procedure were based on an experiment by Craik and Birtwistle (1971). Items were presented orally at a $2-\mathrm{sec}$ rate, with $60 \mathrm{sec}$ allowed for written free recall. In an effort to hold Ss' strategies constant, they were asked to recall words at the end of the input list first. Correctly recalled items were credited to one of two tallies; items probably retrieved from primary memory (PM) and items probably retrieved from secondary memory (SM). Following Tulving and Colotla (1970), PM and SM were defined in terms of intratrial repetition interval (ITRI), i.e., the number of presentations and recalls of other items intervening between presentation and recall of any given item. (For example, if following presentation of a list $\mathrm{ABCDE}$, a $S$ recalled $E, C$, and $D$ in that order, the ITRIs for these items would be 0,3 , and 3 , respectively.) Words with an ITRI of seven or less were tallied as having been retrieved from PM; other recalls were credited to SM.

\section{Results}

The mean number of words recalled per trial was 7.1 $(\mathrm{SD}=1.6)$, of which $2.7(\mathrm{SD}=0.8)$ were in $\mathrm{PM}$ and 4.4 $(\mathrm{SD}=1.9)$ were in $\mathrm{SM}$. Separate analyses of variance were performed on the PM and SM scores. The number of items retrieved from PM was not a function of trials, lists, or instructions. For SM scores, there were significant main effects of trials $[F(4,224)=13.1$, $\mathrm{p}<.001]$ and of lists $[\mathrm{F}(1,56)=26.0, \mathrm{p}<.001]$ but not of instructions $[F(1,56)=0.7]$. There was also a significant Trials by Lists interaction $[F(4,224)=3.8$. $p<.01]$. Table 1 shows that the groups were well matched on Trial 1 but that they diverged progressively thereafter. The control Ss showed a marked PI effect. The experimental Ss showed little or none; in a separate analysis of experimental Ss' scores, the trials effect was not statistically significant $[F(4,116)=1.4]$.

\section{EXPERIMENT II}

\section{Design and Procedure}

A second experiment was conducted to check on the main findings of Experiment 1, using lists drawn from another category (animals); a "release" trial was included to see whether recall of words from a second category would be affected by the way in which first-category lists had been organized. The Ss were presented with four 15-item lists of animals followed by one of occupations. Experimental Ss received the experimental lists of animals, control Ss the control lists of animals; all Ss received one of the five control lists of occupations from Experiment I. All Ss were informed that they would hear four lists of animals and one of occupations. A total of 30 Ss ( 15 experimental, 15 control) from the same source as Experiment I Ss was tested. Details of the procedure and scoring were the same as in Experiment I.

\section{Results}

Once again the number of items retrieved from PM (mean $=2.5, \mathrm{SD}=1.0$ ) was not a function of trials or of lists. Analysis of variance on the SM scores for Trials 1-4 revealed the same pattern as in Experiment 1 . The main effect of trials $[F(3,84)=7.5, p<.001]$ and of lists $[F(1,28)=19.4, p<.001]$ and the Trials by Lists interaction $[F(3,84)=4.8, p<.01]$ were significant. The groups were well matched on Trial 1 (see Table 1). On Trials 2-4 the control Ss showed a marked PI effect, while the experimental Ss showed little or none; a separate analysis of the experimental Ss' scores yielded a nonsignificant trials effect $[F(3,42)=0.4]$. The difference between the groups disappeared on Trial 5 (the "release" trial), where their performance was virtually identical.

\section{DISCUSSION}

The results of both experiments confirm the view of Craik and Birtwistle (1971) that the PI effect is a phenomenon of SM rather than PM.

The performance of the control Ss demonstrates that PI can occur if successive lists share the feature "members of the category of occupations (or animals)." The experimental lists also share this feature, but in their case it is not sufficient to produce a decrement in recall performance across trials. This finding is not, perhaps, very surprising; but it may contribute toward a precise statement of the conditions under which PI will be found. It would appear that, when the set of items on any one list is more homogeneous than the set of items 
from all lists taken together, the PI effect will be attenuated. In the present case, the fact that successive lists drew upon the same category was neutralized by the fact that they also drew upon discrete subcategories. The subcategories, as it were, functioned like categories, with the result that every trial was a "release" trial.

If subcategories do indeed function like categories, one would expect that PI (built up over several lists drawn from the same subcategory) would be released by switching to a list drawn from a second subcategory. Were release from PI not to occur in such circumstances, the PI would have to be attributed to the shared category membership rather than to the shared subcategory membership, i.e., to the second lowest rather than to the lowest between-list shared feature. Gardiner, Craik, and Birtwistle (1972) conducted such an experiment, using "flowers" (or "sports") as the category with "garden" and "wild" ("indoor" and "outdoor") as subcategories. While PI built up over three lists from one subcategory, presentation of a fourth list from the other subcategory did not result in release from PI unless the second subcategory was explicitly labeled for the Ss. Why the release effect failed to occur in the Gardiner et al experiment is unclear. It may be that Ss failed to detect that all items in Lists 1-3 shared șubcategory as well as category membership; each list comprised only three items. It may well be that the lowest shared feature, either within or between lists, is not automatically perceived as such by Ss and that it is perceived shared features that are of functional importance. In this case, it will be necessary to establish what factors determine the salience of shared features. List length may well be such a factor.

The object of the Gardiner et al experiment was to pin down the locus of the PI effect; the critical processes might occur at encoding, during storage, or at retrieval. While the present experiments were designed on the basis of a rationale that assumes encoding is the critical process, the findings are compatible with any locus that has been suggested.

In Experiment II, both groups did equally well on the "release" trial. If it is accepted that no PI had built up in the experimental Ss, it would appear that the release from PI in the control Ss was complete. Certainly, they recalled markedly more items than control $\mathrm{Ss}$ in Experiment I did on Trial 5. But the Ss in Experiment II also recalled more occupations on their release trial than the comparable (i.e., control) Ss in Experiment I did on their first trial $[\mathrm{t}(58)=4.5, \mathrm{p}<.001]$. This is puzzling, since it leads to the conclusion that we have obtained more than $100 \%$ release from PI. Whether there is a genuine enhancement of recall on the release trial is not clear. Perhaps we are using an inappropriate method for quantifying release from PI. The fact that occupations appear to be harder to recall than animals complicates the task of interpretation.

\section{REFERENCES}

Craik, F. I. M., \& Birtwistle, J. Proactive inhibition in free recall. Journal of Experimental Psychology, 1971, 91, 120-123.

Gardiner, J. M., Craik, F. I. M., \& Birtwistle, J. Retrieval cues and release from proactive inhibition. Journal of Verbal Learning \& Verbal Behavior, 1972, 11, 778-783.

Reutener, D. B. Class shift, symbolic shift, and background shift in short-term memory. Journal of Experimental Psychology, $1972,93,90-94$.

Tulving, E., \& Colotla, V. A. Free recall of trilingual lists. Cognitive Psychology, 1970, 1, 86-98.

Wickens, D. D. Encoding categories of words: An empirical approach to meaning. Psychological Review, 1970, 77, 1-15.

Wickens, D. D., \& Clark, S. E. Osgood dimensions as an encoding, class in short-term memory. Journal of Experimental Psychology, 1968, 78, 580-584.

(Revision received July 5, 1973; accepted July 9, 1973.) 